\title{
AUTHOR INDEX Volume 13
}

Abdel-Wanis, M. E., Solyman, M. T. M. and Ali Hasan, N. M., MRI Pattern of Vertebral Fractures Due to Osteoporosis, Infection, and Malignant Tumors

Acharya, K. K. V., Pandey, V. and Rao, P. S., Knee Dislocation with Multi-Ligament Injury: Evaluation, Treatment and Results

Adedoyin, R. A., see Mbada, C. E.

Adegoke, B. O. A., see Bello, A. I.

Aggarwal, A., see Pawar, I.

Aggarwal, A., see Kumar, S.

Aggarwal, A., Kumar, S. and Kumar, D., Effect of Core Stabilization Training on the Lower Back Endurance in Recreationally Active Individuals

Agyepong-Badu, S., see Bello, A. I.

Ali Hasan, N. M., see Abdel-Wanis, M. E.

Almusa, E., see Siddiqui, N. A.

Ando, K., Matsuyama, Y., Sakai, Y., Imagama, S., Ito, Z., Wakao, N. and Ishiguro, N., Cervical Intramedullary Glioblastoma with Intracranial Dissemination: Description of a Rapidly Progressing Case and a Literature Review

Applegarth, S. P., see Haiduven, D. J.

Ayanniyi, O., see Mbada, C. E.

Bello, A. I., Kalu, N. H., Adegoke, B. O. A. and Agyepong-Badu, S., Hydrotherapy versus Land-Based Exercises in the Management of Chronic Low Back Pain:

A Comparative Study

Bhat, A. K., see Eapen, C.

Britton, C. A., see Siddiqui, N. A.

Bugga, P., see Pawar, I.

Caban-Martinez, A. J., Lee, D. J., Clarke, T. C., Davila, E. P., Clark III, J. D., Ocasio, M. A. and Fleming, L. E., Self-Reported Joint and Back Pain Among Hispanic

Construction Workers: A Pilot Workplace Musculoskeletal Assessment

Chang, W.-C., see Chen, S.-Y.

Chan, C., Sihler, E., Kijek, T., Miller, B. S. and Hughes, R. E., A Mobile Computing Tool for Collecting Clinical Outcomes Data from Shoulder Patients

Chao, S.-C., see Chen, S.-Y.

Charpak, G., see Dubousset, J.

Chen, S.-Y., Chao, S.-C., Chang, W.-C., Shen, C.-C. and Tsou, H.-K., Primary Thoracic Intraspinal Hemangiopericytoma Mimics Meningeal Tumor: A Case Report and Literature Review
$13(2010) 57-63$

13 (2010) 119-126

13 (2010) 13-21

13 (2010) 159-165

13 (2010) 153-157

13 (2010) 177-185

13 (2010) 167-176

13 (2010) 159-165

13 (2010) 57-63

13 (2010) 147-151

13 (2010) 43-48

13 (2010) 65-74

13 (2010) 13-21

13 (2010) 159-165

13 (2010) 137-145

13 (2010) 147-151

13 (2010) 153-157

13 (2010) 49-55

13 (2010) 83-88

13 (2010) 127-136

13 (2010) 83-88

13 (2010) 1-12

13 (2010) 83-88 
Chikenji, T., Toda, H., Gyoku, C., Oikawa, N., Katayose, M. and Tsubota, S., A Comparison of the Strength of the Abduction of the Little and Index Fingers and Palmar Abduction and Opposition of the Thumb Between College Baseball Players and Inexperienced Sports Players

Clark III, J. D., see Caban-Martinez, A. J.

Clarke, T. C., see Caban-Martinez, A. J.

Cui, X., see Li, X.

Davila, E. P., see Caban-Martinez, A. J.

Deguise, J., see Dubousset, J.

Downie, B. K., see Scibek, J. S.

Dubousset, J., Charpak, G., Skalli, W., Deguise, J. and Kalifa, G., EOS: A New Imaging System with Low Dose Radiation in Standing Position for Spine and Bone \& Joint Disorders

Eapen, C., Kumar, B. and Bhat, A. K., Prevalence of Cumulative Trauma Disorders in Cell Phone Users

Felson, D. T., see Shamir, L.

Fenichel, I., Salai, M. and Velkes, S., An Acute Fracture of the Femoral Neck Complicating Bone Marrow Edema in Osteonecrosis of the Femoral Head, Treated with Total Hip Replacement

Fenichel, I. and Velkes, S., Bone-Preserving HIP Arthroplasties as an Alternative to Conventional HIP Replacement for Young Patients - A Review Article

Ferrucci, L., see Shamir, L.

Fleming, L. E., see Caban-Martinez, A. J.

Friedman, S. M., see Gonzales Chaves, M. M. S.

Goldberg, I. G., see Shamir, L.

Gonzales Chaves, M. M. S., Marote, C., Pellegrini, G. G., Pighin, A., de Landeta, M. C., Lifshitz, F., Friedman, S. M., Mandalunis, P. and Zeni, S. N., Bone Mass Recovery of Osteopenic Vitamin D Insufficient Rats from Strontium Ranelate Treatment: Does the Response Depend on Vitamin D Nutritional Status or on Source of Vitamin D (D2 Versus D3)?

Gyoku, C., see Chikenji, T.

Haiduven, D. J., Applegarth, S. P., Mcguire-Wolfe, C. and Tenouri, M., Automated and Manual Measurement of the Forces Required to Use Retractable Intramuscular Syringes

He, C., see Zhang, J.

He, H., see Zhang, J.

Hughes, R. E., see Scibek, J. S.

Hughes, R. E., see Chan, C.

Imagama, S., see Ando, K.

Ishiguro, N., see Ando, K.

Ito, Z., see Ando, K.

Johnson, O. E., see Mbada, C. E.

Kalifa, G., see Dubousset, J.

Kalu, N. H., see Bello, A. I.

Katayose, M., see Chikenji, T.

Kijek, T., see Chan, C.

Kumar, B., see Eapen, C.

Kumar, D., see Aggarwal, A.

13 (2010) 75-82

13 (2010) 49-55

13 (2010) 49-55

13 (2010) 203-207

13 (2010) 49-55

13 (2010) 1-12

13 (2010) 23-33

13 (2010) 1-12

13 (2010) 137-145

13 (2010) 197-201

13 (2010) 39-42

13 (2010) 89-94

13 (2010) 197-201

13 (2010) 49-55

13 (2010) 95-108

13 (2010) 197-201

13 (2010) 95-108

13 (2010) 75-82

13 (2010) 65-74

13 (2010) 35-37

13 (2010) 35-37

13 (2010) 23-33

13 (2010) 127-136

13 (2010) 43-48

13 (2010) 43-48

13 (2010) 43-48

13 (2010) 13-21

13 (2010) 1-12

13 (2010) 159-165

13 (2010) 75-82

13 (2010) 127-136

13 (2010) 137-145

13 (2010) 167-176 
Kumar, S., see Aggarwal, A.

13 (2010) 167-176

Kumar, S., Sharma, V. P. and Aggarwal, A., Correlation and Regression Among Pain, Physical Strength, Functional Ability, Quality of Life and Sexual Frequency

in Low Back Pain

de Landeta, M. C., see Gonzales Chaves, M. M. S.

Lee, D. J., see Caban-Martinez, A. J.

Li, X., Zhang, J., Cui, X., Zhao, L., Shen, P. and Zhao, L., Congenital Neuromuscular

Disease with Uniform Type-1 Fibers Simultaneously Presenting Gluteal Muscles

Contracture and Brain Atrophy: A Case Report

Lifshitz, F., see Gonzales Chaves, M. M. S.

Lin, H., see Zhang, J.

Luo, Q., see Zhang, J.

Machado, M., see Silva, L. E.

Mandalunis, P., see Gonzales Chaves, M. M. S.

Marote, C., see Gonzales Chaves, M. M. S.

Matsuyama, Y., see Ando, K.

Mbada, C. E., Ayanniyi, O., Adedoyin, R. A. and Johnson, O. E., Static Endurance of the Back Extensor Muscles: Association Between Performance and Reported Reasons for Test Termination

Mcguire-Wolfe, C., see Haiduven, D. J.

Mell, A. G., see Scibek, J. S.

Miller, B. S., see Chan, C.

Mittal, A., see Pawar, I.

Ocasio, M. A., see Caban-Martinez, A. J.

Oikawa, N., see Chikenji, T.

Palmieri-Smith, R., see Scibek, J. S.

Pandey, V., see Acharya, K. K. V.

Pawar, I., Mittal, A., Bugga, P. and Aggarwal, A., Large Hydatid Cyst in Thigh: A Rare Case with Clinico-Radio-Pathological Profile

Pellegrini, G. G., see Gonzales Chaves, M. M. S.

Pereira, R., see Silva, L. E.

Pighin, A., see Gonzales Chaves, M. M. S.

Rao, P. S., see Acharya, K. K. V.

Rodrigues, V. C., see Silva, L. E.

Sakai, Y., see Ando, K.

Salai, M., see Fenichel, I.

Sandhu, J. S., Trivedi, S. S. and Shenoy, S., A Correlation Study Between Plasma Myostatin and Peak Torque Strength Gains of Bilateral Knee Extensors and Flexors after Resistance Training in Healthy Asian Indians

13 (2010) 177-175

13 (2010) 95-108

13 (2010) 49-55

13 (2010) 203-207

13 (2010) 95-108

13 (2010) 35-37

13 (2010) 35-37

13 (2010) 187-195

13 (2010) 95-108

13 (2010) 95-108

13 (2010) 43-48

13 (2010) 13-21

13 (2010) 65-74

13 (2010) 23-33

13 (2010) 127-136

13 (2010) 153-157

13 (2010) 49-55

13 (2010) 75-82

13 (2010) 23-33

13 (2010) 119-126

13 (2010) 153-157

13 (2010) 95-108

13 (2010) 187-195

13 (2010) 95-108

13 (2010) 119-126

13 (2010) 187-195

13 (2010) 43-48

13 (2010) 39-42

13 (2010) 109-117

Scibek, J. S., Mell, A. G., Downie, B. K., Palmieri-Smith, R. and Hughes, R. E., Impact of Pain on Shoulder Elevation Velocity in Patients with Rotator Cuff Tears

$13(2010) 23-33$

Shamir, L., Felson, D. T., Ferrucci, L. and Goldberg, I. G., Assessment of Osteoarthritis Initiative-Kellgren and Lawrence Scoring Projects Quality Using Computer Analysis

13 (2010) 197-201

13 (2010) 177-185

13 (2010) 83-88

13 (2010) 203-207

13 (2010) 109-117 
Siddiqui, N. A., Almusa, E. and Britton, C. A., Cervical Spine Pneumatocyst: Case Report and Review of the Literature

13 (2010) 147-151

Sihler, E., see Chan, C.

Silva, K. M., see Silva, L. E.

Silva, L. E., Pereira, R., Rodrigues, V. C., Silva, P. A. V., Silva, K. M. and Machado, M., 13 (2010) 127-136

13 (2010) 187-195 Length of Rest Interval Between Resistance Exercise Sets: Performance and Inter-Individual Variability of CK Activity

Silva, P. A. V., see Silva, L. E.

Skalli, W., see Dubousset, J.

Solyman, M. T. M., see Abdel-Wanis, M. E.

Tenouri, M., see Haiduven, D. J.

Toda, H., see Chikenji, T.

Trivedi, S. S., see Sandhu, J. S.

Tsou, H.-K., see Chen, S.-Y.

Tsubota, S., see Chikenji, T.

Velkes, S., see Fenichel, I.

Velkes, S., see Fenichel, I.

Wakao, N., see Ando, K.

Zeni, S. N., see Gonzales Chaves, M. M. S.

Zhang, J., see Li, X.

Zhang, J., He, H., Lin, H., Luo, Q. and He, C., To Increase Risk Awareness and Avoid Rehabilitation Treatment Risk: A Case Report of the Cervical Spine Disease Associated with Intracranial Arterial Aneurysm Diagnosis and Treatment

13 (2010) 187-195

13 (2010) 187-195

13 (2010) $1-12$

13 (2010) 57-63

13 (2010) 65-74

13 (2010) 75-82

13 (2010) 109-117

13 (2010) 83-88

13 (2010) 75-82

13 (2010) 39-42

13 (2010) 89-94

13 (2010) 43-48

13 (2010) 95-108

13 (2010) 203-207

Zhao, L., see Li, X.

13 (2010) 35-37

13 (2010) 203-207 